\title{
Zerfall und Verjüngung eines Schutzwaldes nach dem Absterben der Fichten durch Buchdruckerbefall
}

\author{
Andrea Doris Kupferschmid*
}

\begin{abstract}
Decay and Tree Regeneration in a Protection Forest after Spruce Die-back Caused by Bark Beetles

Abstract: After the storm "Vivian" in 1990, bark beetle infestations caused the dieback of adjacent Norway spruce stands, such as on the Gandberg mountain forest in the Swiss Alps. Field studies were conducted in these steep snag stands to describe the short-term succession, and a mathematical model focusing on tree regeneration was used to project the long-term development. Seventy-five percent of all snags were broken 8 years after the die-back, and the ground vegetation changed to raspberry brush or it became dominated by mosses, ferns and grasses. Assuming only a moderate browsing pressure in the model, the simulations together with the investigations on snag decay and the height of piled up logs suggest that the protective effect against avalanches and rockfall will first be maintained by the snags, stumps and logs, then by both the timber and the new Picea regeneration, and finally by the new Picea trees. Thus, leaving snag stands uncleared can result in effective protection for several decades.
\end{abstract}

Keywords: bark beetle, coarse woody debris, ecological modelling, protection forest, succession, tree regeneration, ungulate browsing

m 27. und 28. Februar 1990 warf der Sturm Vivian rund 4.9 Millionen Kubikmeter Holz im Schweizer Wald; mit etwa 343000 Kubikmeter geworfenem Holz war der Kanton Glarus eine der am stärksten betroffenen Regionen. Das nach dem Sturm liegen gebliebene Holz bot dem Buchdrucker (Ips typographus), dem häufigsten Borkenkäfer, der vor allem Fichten befällt, gutes Brutmaterial. Die folgenden trockenen und warmen Jahre begünstigten zusätzlich die Massenvermehrung dieser Käfer. Dies führte dazu, daß lebende Fichten in angrenzenden, geschwächten Wäldern ebenfalls von diesen gefräßigen Borkenkäfern befallen wurden und sodann abstarben. Entsprechend fiel dem Buchdrucker im Kanton Glarus mit 321866 Kubikmetern eine etwa gleich große Holzmenge zum Opfer wie zuvor dem Sturm Vivian. Beispielsweise wurden in den Fichtenwäldern oberhalb des Dorfes Schwanden zunächst 154 Hektar Wald vom Sturm geworfen; zwischen 1992 und 1997 starben dann durch Buchdruckerbefall die Fichten in den angrenzenden Wäldern auf einer Fläche von mehr als 100 Hektar ab (Figur 1). Als Folge blieben am

* Postadresse: Dr. A.D. Kupferschmid Albisetti Fadacherstr. 17

CH-8340 Hinwil (Schweiz)
Gandberg Wälder aus stehenden toten Fichten zurück, sogenannte Totholzbestände. Der Forstdienst räumte zwar den vom Sturm direkt oberhalb des Dorfes geworfenen Schutzwald (d. h. einen Wald, der Siedlungen und Verkehrswege vor Lawinen und Steinschlag schützt). Nicht geräumt wurden jedoch die durch Buchdruckerbefall entstandenen Totholzbe- stände am benachbarten Gandberg in einer Höhe von 1200-1600 Meter über dem Meeresspiegel (ü. d. M), da diese nur schwer zugänglich waren und hohe Räumungskosten verursacht hätten. 1996 wurde der Gandbergwald zum Naturwaldreservat erklärt. Der Entscheid, diese Totholzbestände stehen zu lassen, war damals mutig. Denn nirgendwo in Europa konnte man auf Erfahrung mit so großen Totholzflächen an steilen Gebirgshängen zurückgreifen - die Kenntnisse beschränkten sich auf Totholzbestände in flachen Hochlagen ohne jegliche Schutzfunktion, wie beispielsweise im Bayerischen Wald ${ }^{[1,2]}$. In unbewaldetem Zustand - zum Beispiel nach der Räumung des Totholzes - würden im steilen Gelände am Gandberg Lawinen anreißen. Außerdem besteht infolge des brüchigen, roten Konglomeratgesteins Steinschlaggefahr. Es stellten sich in bezug auf die mögliche Schutzfunktion von Totholzbeständen daher vor allem zwei Fragen:

Kann ein Totholzbestand, ähnlich wie vorher der lebende Fichtenbestand, das Anreißen von Waldlawinen verhindern und Steinschläge abbremsen?

Wie lange dauert es, bis die Baumverjüngung in einem solchen Totholzbestand zu einem schutzfähigen Bestand herangewachsen ist?

Der Gandbergwald war als erstes großes Studienobjekt optimal gelegen, um diese Fragen $\mathrm{zu}$ beantworten. Indem er Steinschlag abbremsen und Lawinenanrisse verhindern kann, gleicht er einem Schutzwald, ohne jedoch eine direkte Schutzfunktion zu haben, da unterhalb des Waldes weder ein bewohntes Dorf noch eine wichtige Straße liegen. Da Feld-

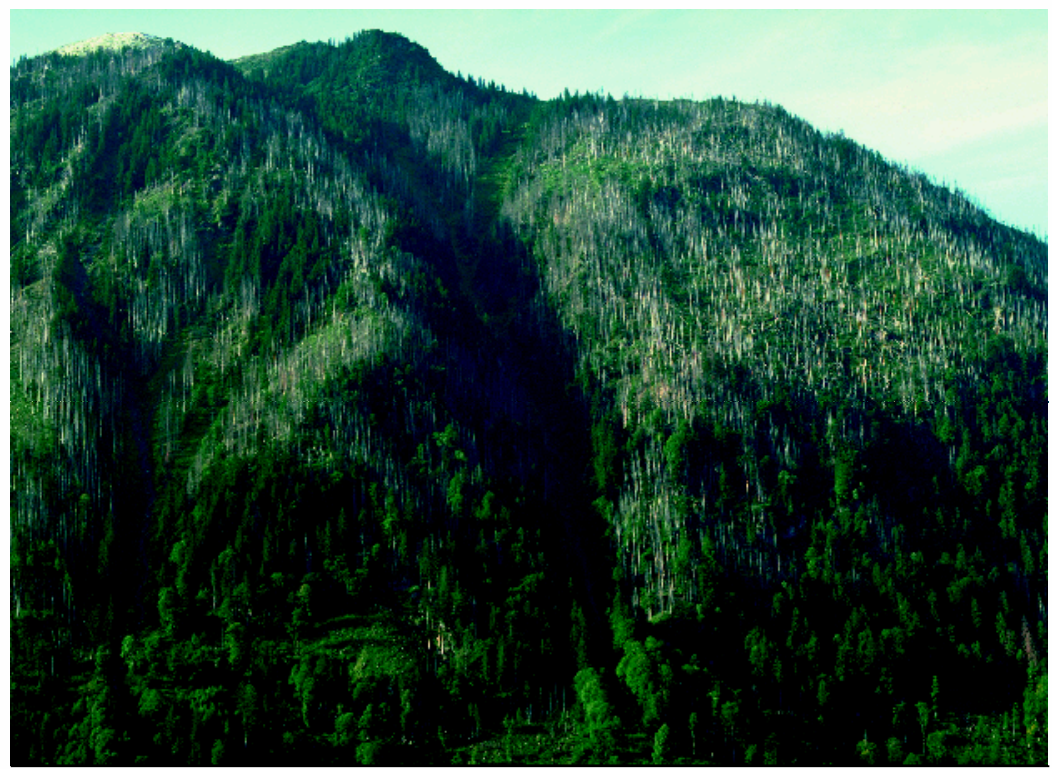


erhebungen nur eine Aussage über den aktuellen Zustand und die bisherige Entwicklung zulassen und Verjüngungsprozesse in einem Gebirgswald sehr langsam vor sich gehen, entwickelte ich ein Modell über den Zerfall und die Verjüngung von Totholzbeständen. Das Modell basiert auf erhobenen Daten aus dem Gandbergwald und auf Literaturdaten. Simulationen über die ersten Jahre wurden mit Felddaten des Jahres 2001 aus dem Gandbergwald verglichen. Das Ziel war, die wahrscheinlichste längerfristige Entwicklung dieser Fichtentotholzbestände an Steilhängen aufzuzeigen und damit ein Instrument zu schaffen, mit dem sich die Verjüngungsprozesse nach Buchdrucker-Massenausbreitungen beurteilen lassen.

\section{Vegetationsentwicklung}

Um die Entwicklung der Vegetation zu verfolgen, wurde seit dem Absterben der Fichten 1993 jährlich in 24 ein mal ein Meter großen Dauerflächen die Vegetation aufgenommen. Zusätzlich wurden im Jahr 2001 entlang von vier horizontalen Streifentransekten (5 Meter breit und 100-160 Meter lang) in der montanen (1200-1450 Meter ü. d. M.) und in der subalpinen (1450 und 1600 Meter ü. d. M.) Höhenstufe des Gandbergwaldes die Anteile der Bedeckung des Bodens durch Vegetation, Stämme und Felsen geschätzt.

In der montanen Höhenstufe war der Waldboden des früher geschlossenen Labkraut-Tannen-Fichtenwaldes (GalioAbieti-Piceetum-Assoziation) locker mit Moosen (Polytrichum formosum, Hylocomium splendens, Dicranum scoparium etc.), Rundblättrigem Labkraut (Galium rotondifolium) und Sauerklee (Oxalis acetosella) überzogen. Diese Arten pro-

Figur 2. Montaner Bereich des Gandbergwaldes mit vielen horizontal liegenden Stämmen und einer üppigen Schlagflora aus Himbeeren. (Photo: A.D. Kupferschmid, Sommer 2000) fitierten von dem in den ersten Jahren nach Absterben der Fichten erhöhten Licht- und Nährstoffangebot und besiedelten zusätzlich vegetationsfreie Stellen. Seit ungefähr 1997 dominierten in der montanen Stufe Himbeeren (Rubus idae$u s$ ), Farne (Dryopteris dilatata, Oreopteris limbosperma und Athyrium filixfemina) und Waldweidenröschen (Epilobium angustifolium) (Figur 2).

In der subalpinen Höhenstufe kamen hingegen bis ins Jahr 2000 nur an wenigen Stellen - so auf Wurzeltellern und teilweise in der Nadelstreu unter den abgestorbenen Fichten - Himbeeren und Waldweidenröschen auf. Von diesen Stellen abgesehen, war die subalpine Vegetationsstruktur des AlpenlattichFichtenwaldes mit Heidelbeere (Homogyno-Piceetum-vaccinietosum-myrtilliAssoziation) weitgehend erhalten geblieben. Farne oder Wolliges Reitgras (Calamagrostis villosa) wuchsen in unbewaldeten Lücken. Nadelstreu oder Haarmützenmoose (Polytrichum formosum und Polytrichum alpinum) fanden sich direkt unter dem früheren Schirm der Fichten. Zwischen diesen Pflanzengemeinschaften wuchs Haarmützenmoos zusammen mit Wald-Sternmiere (Stellaria nemorum), Behaarter Hainsimse (Luzula pilosa), Waldhainsimse (Luzula sylvatica), Bräunlicher Segge (Carex brunnescens), Waldsegge (Carex flacca) und Draht-Schmiele (Avenella flexuosa) (Figur 3).

\section{Entwicklung der Baumverjüngung}

In den Dauerflächen wurde außerdem jährlich die Anzahl der gekeimten Bäumchen - die natürliche Baumverjüngung und ihr Höhenzuwachs gemessen. Fich-

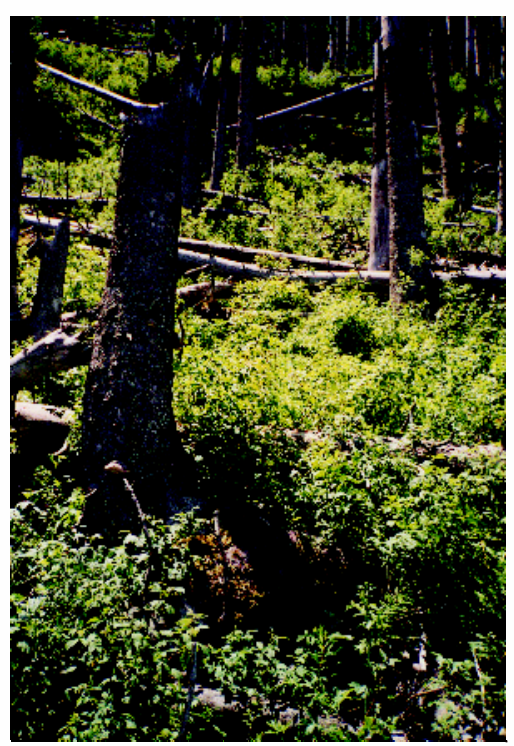

ten (Picea abies) keimten am häufigsten (etwa drei Fichtensämlinge pro Quadratmeter), gefolgt von Bergahorn (Acer pseudoplatanus), Vogelbeere (Sorbus aucuparia), Birke (Betula pendula) und Tanne (Abies alba). Die jährliche Sterberate der Fichtenverjüngung lag, wahrscheinlich infolge der dichten Vegetation, konstant bei ungefähr 25 Prozent, so daß im Jahr 2001 nur noch 0.5 Fichtensämlinge pro Quadratmeter lebten. Obwohl in den Totholzbeständen des Gandbergwaldes einzelne lebende Tannen vorhanden sind, überlebte bis 2001 keine junge Tanne, dies aufgrund des stetigen Verbisses durch Gemsen, welche in diesem Teil des Wildschutzgebietes Freiberg Kärpf sehr häufig vorkommen. Da keine Baumverjüngung aus der Zeit vor dem Absterben der Fichten existierte und da die jungen Bäumchen häufig verbissen wurden, waren die Fichten und Bergahorne im Jahr 2001 im Mittel erst 13 Zentimeter hoch. Lediglich einzelne Birken hatten, da sie weniger durch Gemsen verbissen werden, zu dieser Zeit eine Höhe von zwei Metern erreicht ${ }^{[3]}$.

\section{Zerfallsdynamik}

In den vier horizontalen Streifentransekten, in denen die Vegetationsdeckung geschätzt wurde, wurden auch alle stehenden toten Bäume, Strünke und das liegende Holz mit einem Brusthöhendurchmesser über 16 Zentimeter vermessen. Das damit errechnete totale Holzvolumen lag im montanen Bereich des Gandbergwaldes bei 800 Kubikmeter pro Hektar und im subalpinen Bereich bei $600 \mathrm{Ku}$ bikmeter pro Hektar. Zusätzlich wurde jeweils geschätzt, wann im Zeitraum zwischen 1993 und 2000 die Stämme abgebrochen waren. Im Sommer 2000 waren 25 Prozent der toten Bäume nicht gebrochen oder wiesen nur Wipfelbrüche auf, 25 Prozent waren in einer Stammhöhe über zehn Meter gebrochen und 50 Prozent unterhalb von zehn Metern. Während heftiger Stürme werden lebende Fichten oft entwurzelt. Die toten Bäume des Gandbergwaldes wurden dagegen nicht einmal während des Sturmes Lothar vom 26. Dezember 1999 entwurzelt, sondern brachen (28 Prozent aller Bäume), meist in einer Baumhöhe von ein bis fünf Metern ${ }^{[4]}$.

Durchschnittlich waren in der montanen Stufe rund 20 Prozent der Bodenoberfläche mit Holz (liegenden Stämmen und Strünken) bedeckt, in der subalpinen 12 Prozent. Die meisten Bäume waren zwischen den Strünken und Stämmen auf einer durchschnittlichen Höhe von 85 Zentimetern über dem Boden verkeilt. Die gebrochenen Stämme lagen im 


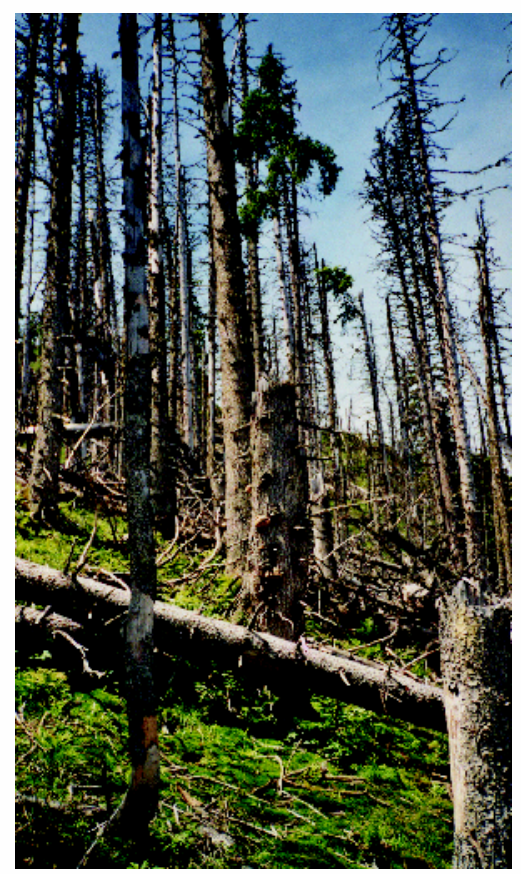

steilen montanen Bereich meist in südöstlicher-östlicher Richtung, also horizontal bis schräg zum Hang ${ }^{[4]}(\mathrm{Fi}$ gur 2). Die liegenden Stämme konnten deshalb Steine abbremsen und teilweise auch zurückhalten. Zusammen mit den liegenden und verkeilten Stämmen verhinderten die stehenden toten Fichten und Strünke den Aufbau einer gleichförmigen Schneedecke und boten damit auch Schutz vor dem Anreißen von Waldlawinen. Wäre das Totholz des Gandbergwaldes geräumt worden, wäre die Gefahr für lokale Bodenerosion, Steinschläge und Lawinenabgänge heute sicher größer als in den derzeitigen Totholzbeständen.

In den nächsten Jahren werden auch die letzten noch stehenden Fichten infolge zunehmender Zersetzung durch Pilze und Insekten abbrechen. Die verkeilten Stämme werden zusammensacken, so daß die Schutzwirkung durch das Totholz abnimmt. In der nahe gelegenen Windwurffläche oberhalb von Schwanden wurde anhand von Stabilitätsanalysen an verkeilten Stämmen geschlossen, daß die liegenden Stämme und Strünke etwa 30 Jahre vor Naturgefahren schützen wer-

\footnotetext{
1) Die Kleinstandortstypen wurden definiert anhand der dominierenden Pflanzenarten und Bodenauflage oder der Zerfallsstruktur (z. B. Himbeergestrüpp, Moderholz oder mit Moosen bewachsener Blockschutt).
}

Figur 3. Der subalpine Bereich des Gandbergwaldes wurde auch in den ersten Jahren nach dem Absterben der Fichten weiterhin von Moosen, Farnen und Reitgras dominiert.

(Photo: A.D. Kupferschmid, Sommer 2000)

den ${ }^{[5]}$. Ähnliches kann für die Totholzbestände des Gandbergwaldes angenommen werden ${ }^{[4]}$.

\section{Modell der Verjüngung von Totholz- beständen}

Mein Modell simuliert das jährliche Aufwachsen der Baumverjüngung. Dazu habe ich die Baumverjüngung in neun Höhenklassen eingeteilt $(<10,10.5-20$, 20.5-40, 40.5-70, 70.5-100, 100.5-130, $130.5-250,250.5-500,>500 \mathrm{~cm})$. Ich gehe davon aus, daß Fichten, die größer als fünf Meter sind, Schutz vor Waldlawinen und Steinschlag bieten. Deshalb ist die Zielvariable meines Modells die Anzahl der Fichten, die größer als fünf Meter sind (Höhenklasse $>5 \mathrm{~m}$ ). Weiter gehe ich davon aus, daß die Keimfähigkeit, das Baumwachstum und die Sterberate bis zu einer Baumhöhe von 130 Zentimetern - entsprechend der durchschnittlichen Höhe der Himbeeren, die mit den aufwachsenden Bäumchen konkurrieren - von den jeweiligen Kleinstandortstypen ${ }^{1)}$ abhängig sind. Aus dem Gandbergwald (siehe Abschnitt "Entwicklung der Baumverjüngung") und Kenntnissen aus der Verjüngungsökologie habe ich die Keimungs-, Wachs- tums- und Sterberaten deshalb für jede Baumart (Fichten, Birken, Bergahorn und Vogelbeere) und für jeden der 26 Kleinstandortstypen spezifisch geschätzt. Die Wachstums- und Sterberaten für Bäume ab einer Höhe von 130 Zentimetern wurden nur noch je Höhenklasse und Baumart geschätzt. Zudem wurden für die montane und für die subalpine Höhenstufe unterschiedliche Anfangswerte (z. B. Samenzahl, vorhandene Kleinstandorte) und Einflußgrößen (z. B. standortsabhängige Keimungs-, Wachstums- und Sterberaten) ins Modell eingesetzt.

Im Laufe der Sukzession - der zeitlichen Abfolge von zum Beispiel Vegetationsmustern - ändern sich innerhalb eines Totholzbestandes auch die Kleinstandortstypen. Diese Änderung beeinflußt, wie oben dargestellt, die Baumverjüngung. Daher umfaßt mein Modell auch die Zerfallsdynamik und Vegetationsentwicklung, das heißt es wird in zwei Submodellen berechnet, wann welcher Kleinstandortstyp wie häufig vorhanden ist. Im Zerfalls-Submodell wird simuliert, wie viele tote Bäume pro Jahr brechen, welche Fläche diese Stämme bedecken (Kleinstandort Stämme) und ab wann auf ihnen eine Baumverjüngung (Moderholzverjüngung) möglich ist. Im Vegetations-Submodell wird die Vegetationsentwicklung vom lebenden Bestand über den Totholzbestand bis hin zu einem neuen Baumbestand mit Hilfe von weiteren Kleinstandortstypen simuliert. Dabei wurde anhand der Felddaten berechnet, mit welcher Wahrscheinlichkeit sich ein Kleinstandortstyp innerhalb eines Jahres zu einem anderen Kleinstandortstyp hin verändert (MatrizenModell) ${ }^{[6]}$.

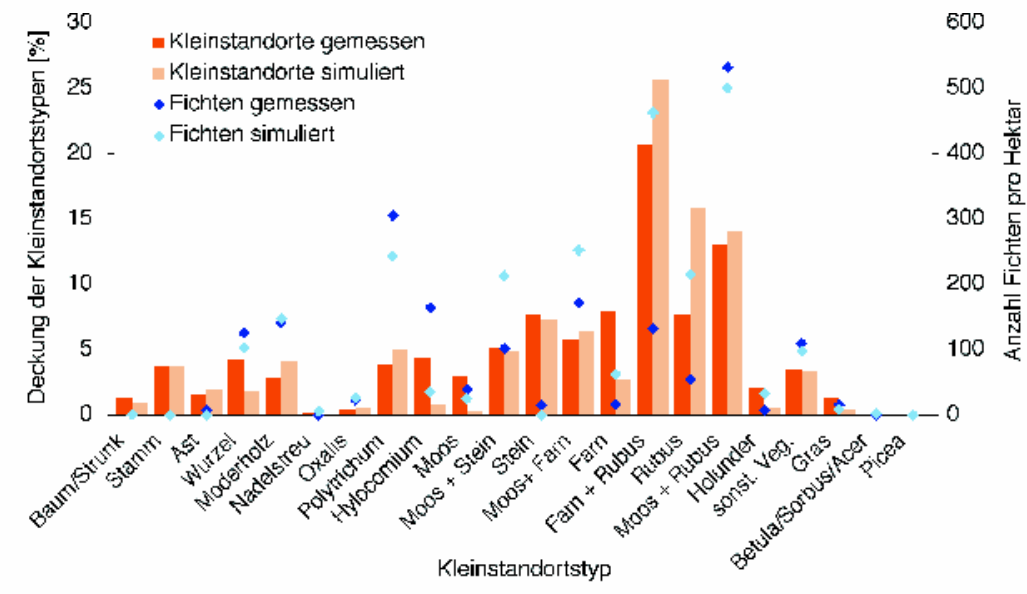

Figur 4. Test des Baumverjüngungsmodells für den montanen Bereich des Gandbergwaldes: Vergleich der gemessenen mit den simulierten Anteilen der verschiedenen Kleinstandortstypen im Jahr 2001 und der gezählten und simulierten Anzahl Fichten im Jahr 2001. 


\section{Simulationen und Überprïfung des Modells}

Im Gandbergwald habe ich entlang von 128 Linien die Häufigkeit der Kleinstandortstypen im Jahre 2001 aufgenommen (Line intercept method). Die Linien waren zehn Meter lang, und je 64 Linien befanden sich auf den beschriebenen zwei Höhenstufen (montan und subalpin). Zusätzlich habe ich die Baumverjüngung innerhalb von zwei Meter breiten Streifen beiderseits dieser Linien gezählt und vermessen.

Ausgehend vom lebenden Bestand 1993 ließ sich die beobachtete Häufigkeit der Kleinstandorte im Jahr 2001 mit den zwei Submodellen für den Zerfall und die Vegetation sehr gut rekonstruieren [6] (Figur 4). Simulationen zeigten, daß die im Feld beobachteten Verjüngungszahlen nur dann mit dem Modell reproduziert werden konnten, wenn kleinstandortsspezifische Keimungs-, Wachstums- und Mortalitätsraten verwendet wurden ${ }^{[7]}$. Langzeitsimulationen ergaben, $\mathrm{da} \beta$ auf dem Gandberg kein geschlossenes Birkenoder Vogelbeerwäldchen entstehen kann, da zu wenige Birken keimten und der Verbiß an den Vogelbeeren zu groß ist. Den Modellberechnungen zufolge wird es nach dem Absterben der Fichten etwa 30-35 Jahre dauern, bis die jungen Fichten (die 1994 und 1996 gekeimt waren) eine Höhe von fünf Meter erreichen. Dabei dürften im montanen Gandbergwald pro Hektar nur etwa 330 dieser Fichten übrig bleiben, während im subalpinen Bereich wahrscheinlich rund 930 Fichten pro Hektar überleben werden ${ }^{[7]}$. Dieser Unterschied ist bedingt durch den sehr hohen winterlichen Wildverbiß im montanen Gandbergwald. Im subalpinen Bereich dürfte die Dichte der jungen Fichten für einen effizienten Schutz vor Lawinenanrissen und eventuell auch vor Steinschlag genügen. Wann in der Zukunft die Verjüngung für eine effektive Schutzwirkung auch im montanen Gandbergwald ausreicht und gut räumlich verteilt sein wird, hängt vor allem von der Wilddichte und der Anzahl Bäumchen ab, die auf den vermodernden Stämmen keimen werden (Moderholzverjüngung).

\section{War der Sturm eine Chance für den Gandbergwald?}

Der Gandbergwald im Kanton Glarus war bis 1993 vor allem in der montanen Höhenstufe ein gleichförmiger Fichtenwald ohne nennenswerte Baumverjüngung. Durch das Absterben der Fichten infolge von Buchdruckerbefall auf einer Fläche von über 100 Hektar verringerte sich das Angebot an Fichtensamen drastisch. Da Fichtensamen vom Wind nur kleine Strecken hangaufwärts transportiert werden, ist es wichtig, die wenigen lebenden Fichten zu erhalten. Gleiches gilt auch für die Bergahorne, Birken, Vogelbeeren und Tannen. Denn dort wo nach dem Zusammenbrechen des Fichtenbestandes keine Bäumchen gekeimt oder die gekeimten Bäumchen abgestorben sind, könnten ohne neue Ansamung größere Lücken im zukünftigen Baumbestand entstehen, womit die Schutzfunktion eventuell in Frage gestellt würde.

Läßt man den Gandbergwald sich selbst erneuern, so wird der aufkommende Bestand in der montanen Höhenstufe in bezug auf das Alter der Bäume wahrscheinlich gemischter sein als der frühere. Dies ist bedingt durch das langsame Vermodern der Stämme und die sich deshalb über lange Zeiträume hinziehende Moderholzverjüngung. Allerdings wird der zukünftige Bestand aufgrund der hohen Wilddichte kaum aus mehr Baumarten bestehen als der frühere. Das Schalenwild, vor allem die Gemse, dürfte Tannen und Bergahorne so stark verbeißen, daß diese absterben, und die Vogelbeeren zumindest stark am Aufwachsen hindern. So bleiben nur Fichten und wenige Birken übrig.

Ohne Sturm und die anschließende Massenvermehrung des Buchdruckers hätte es die Totholzbestände am Gandberg nicht gegeben. Nur aufgrund der großen Mengen an Sturm- und Käferholz, die in dieser Region innerhalb von wenigen Jahren anfielen (die einer mehrfachen Jahresnutzung entsprachen), verzichtete der Forstdienst auf eine Nutzung des Totholzes. So entstand mit dem Gandberg eine der größten Flächen mit stehendem Totholz in der Schweiz. Nach dem Sturm Lothar 1999 gewann der Gandbergwald zusätzlich an Bedeutung: Forstleute aus sturmgeschädigten Gebieten der ganzen Schweiz besichtigten ihn, um den Zerfall und die beginnende Regeneration von Fichtentotholzbeständen nach Buchdrukkerbefall zu verfolgen. Die längerfristige Entwicklung von Totholzbeständen kann in der Natur noch nicht beobachtet werden, jedoch ist mein Modell in der Lage, die wahrscheinlichste Entwicklung der Verjüngung aufzuzeigen. Anhand der Simulationen und der Entwicklung des bisherigen Totholzzerfalls kann davon ausgegangen werden, daß die Schutzwirkung des Gandbergwaldes zuerst durch die stehenden toten Fichten, die Strünke und liegenden Stämme gewährleistet bleibt, danach durch das Totholz und die aufkommenden Fichten und später nur noch durch die neu aufwachsenden Fichten. Die Studie zeigt, daß ungeräumte Totholzbestände an steilen Gebirgslagen über mehrere Jahrzehnte vor Naturgefahren schützen können, sofern der Wildverbiß, wie im subalpinen Gandbergwald, nicht zu hoch ist. Das Belassen von Totholzbeständen in Fichtenschutzwäldern kann deshalb eine sinnvolle ManagementOption sein.

\section{Danksagung}

Die vorliegende Arbeit entstand im Rahmen einer Dissertation unter der Leitung von Prof. H. Bugmann, Dr. W. Schönenberger und Dr. P. Brang an der Eidgenössischen Forschungsanstalt WSL und der ETH Zürich. Finanziert wurde das Projekt durch den Kanton Glarus, den Fonds für Wald- und Holzforschung und die Eidgenössische Forschungsanstalt WSL.

\section{Literaturverzeichnis}

[1] M. Heurich: Waldentwicklung im Bergwald nach Windwurf und Borkenkäferbefall, Nationalparkverwaltung Bayerischer Wald, Grafenau (2001).

[2] M.L. Bauer: Walddynamik nach Borkenkäferbefall in Hochlagen des Bayerischen Waldes, Dissertation, Lehrstuhl für Waldbau und Forsteinrichtung, Technische Universität München, München (2002).

[3] A.D. Kupferschmid, W. Schönenberger, U. Wasem: "Tree regeneration in a Norway spruce snag stand after tree die-back caused by Ips typographus", Forest Snow and Landscape Research 77 (2002) 149-160.

[4] A.D. Kupferschmid Albisetti, P. Brang, W. Schönenberger, H. Bugmann: "Decay of Picea abies snag stands on steep mountain slopes", Forest Chronicle 79 (2003) 247-252.

[5] W. Frey, P. Thee: "Avalanche protection of windthrow areas: A ten year comparison of cleared and uncleared starting zones", Forest Snow and Landscape Research 77 (2002) 89-107.

[6] A.D. Kupferschmid, H. Bugmann: "Predicting decay and vegetation development in Picea abies snag stands", Plant Ecology, submitted.

[7] A.D. Kupferschmid, P. Brang, W. Schönenberger, H. Bugmann: "Predicting tree regeneration in Picea abies snag stands", Forest Science, submitted.

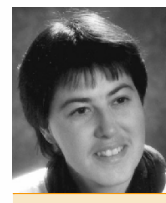

Andrea D. Kupferschmid Albisetti: Geboren 1973 in Luzern. Sie studierte Biologie in Bern und Neuenburg und arbeitete anschließend ein halbes Jahr als Assistentin am Forstwissenschaftlichen Departement der ETH Zürich. Ihre Doktorarbeit zum Thema "Succession in a protection forest after Picea abies die-back" erarbeitete sie bei der Professur für Gebirgswaldökologie der ETH Zürich und im Team Gebirgswaldökologie der Eidgenössischen Forschungsanstalt WSL in Birmensdorf. 